\title{
Evidências da técnica de liberação miofascial no tratamento fisioterapêutico: revisão sistemática
}

\author{
Evidence of myofascial release in the physiotherapeutic treatment: systematic review
}

\author{
Anna Paula Martins ${ }^{1}$ \\ Kamilla Prado Pereira ${ }^{1}$ \\ Lilian Ramiro Felício ${ }^{1,}$
}

\section{Resumo}

Objetivo: analisar os métodos de liberação miofascial, verificando o nível de evidência destes. Métodos: Foi realizada uma busca nas bases de dados SciELO, PubMed e PEDro,utilizando as palavraschaves:myofascial release, physical therapy, manual therapy, sendo os critérios de inclusão: Ensaios Clínicos Randomizados (ECR); idioma inglês ou português, e classificação maior ou igual a 7/10 (escala PEDro), sendo a busca realizada no período de 2008-2018. Resultados:Foram selecionados 132 ECR, dos quais foram excluídos: 27 duplicatas, 28pela análise do título, 11pós análise do resumo, 56 pelaclassificação inferior a 7 na escala PEDro e 3 em decorrência ao idioma, sendo dessa forma, selecionados 7 artigos para a revisão. Os estudos analisados no artigo compararam técnicas de liberação miofascial manual e instrumental, sendo a compressão isquêmica de pontos gatilhos a mais utilizada. Em comparativos entre as técnicas instrumentais, Foam Roll e Fascial Abrasion, a técnica utilizando o Foam Roll se mostrou menos efetiva. Conclusão: Em todas as suas formas de aplicação, a liberação miofascial se mostrou efetiva quanto ao alívio de dor e tensão.

Palavras-Chave: liberação miofascial, modalidades de fisioterapia, reabilitação.

\begin{abstract}
Objective: to analyze the methods of myofascial release, verifying the level of evidence. Methods: A search was performed on the databases SCIELO, PUBMed and PEDro, using the keywords: myofascial release, physical therapy, manual therapy; and inclusion criteria: Randomized Clinical Trials (RCTs); English or Portuguese language, and a rating of 7/10 (PEDro scale), during 2008-2018 September. Results: A total of 132 RCTs were selected, of which 27 were duplicates, 28 were eliminated by title analysis, 11 eliminated after abstract analysis, 56 were eliminated by less 7 on the PEDro scaleclassification and 3 RCTs eliminated by language, so were selected 7 papers for review. The studies analyzed in the article compared techniques of manual and instrumental myofascial release, with ischemic compression of trigger points being the most used. In comparisons between the instrumental techniques, Foam Roll and Fascial Abrasion, the technique using Foam Roll proved to be less effective. Conclusion: In all its forms of application, the myofascial release was effective in relieving pain and tension.
\end{abstract}

Keywords: myofascial release, physical therapy modalities, rehabilitation.

\section{Afiliação dos autores}

${ }^{1}$ Curso de Fisioterapia, Faculdade de Educação Física e Fisioterapia, Universidade Federal de Uberlândia, Uberlândia; Minas Gerais, Brasil.

\section{${ }^{\star}$ Autor correspondente}

Faculdade de Educação Física e Fisioterapia, Universidade Federal de Uberlândia, Rua Benjamin Constant, 1286, CEP: 38400-678 Uberlândia, Minas Gerais, Brasil e-mail: lilianrf@ufu.br

\section{Conflito de interesses}

Os autores declararam não haver conflito de interesses.

\section{Processo de arbitragem}




\section{Introdução}

A fáscia é um tecido conjuntivo propriamente dito, denso, de caráter ininterrupto, que circunda e conecta os músculos, estruturas nervosas e vísceras, sendo um importante elemento de comunicação mecânica entre os vários sistemas corporais ${ }^{1}$. Este tecido tem como principal função realizar a transmissão de força tensional, sendo que o sistema de forças é originado de sua função de revestir e conectar estruturas ${ }^{2}$. Dada a sua característica de alta inervação e células responsáveis por oferecer noção de pressão e estiramento, este tecido frequentemente está relacionado a queixas álgicas e, além disso, colabora na percepção postural ${ }^{3}$.

A transmissão de forças da fáscia é importante nas funções proprioceptivas e nociceptivas ${ }^{4}$. $\mathrm{O}$ conjunto das fáscias geram um componente de biotensegridade, processo em que ocorre a transmissão de forças de modo a estabilizar estruturas, dissipando a carga de forma homogênea pelo corpo. Graças a essa propriedade, a fásciaauxiliana estabilização tecidual, à medida que envolve as estruturas, assumindo um papel estabilização dinâmica do sistema musculoesquelético ${ }^{4}$.

De acordo com Zullo et al. ${ }^{5}$, a fáscia juntamente com os tecidos musculares e conectivos, constitui a maioria dos tecidos acometidos por lesões durante a prática de atividades esportivas, e estas lesões estão relacionadas aos microtraumas contínuos ou de um grande traumatismo tecidual, que resultam em um processo inflamatório, desorganizando esta malha e formando aderências e fibroses ${ }^{6}$.

A Síndrome da Dor Miofascial (SDM) é uma das causas mais comuns de dor musculoesquelética, sendo caracterizada por uma alteração regional neuromuscular e pela presença de locais com aumento de tensão muscular, gerandodor em queimação, sensação de "peso", dor em pontadas, diminuição da força muscular, limitação da amplitude de movimento e, em alguns casos, fadiga muscular, produzindo dor referida em áreas distantes ou adjacentes ${ }^{7}$.

Diversas alterações podem causar a SDM, tais como: processos degenerativos, inflamatórios, macro ou microtraumáticos de inúmeras estruturas, principalmente nas regiões cervicais, cintura escapular e lombar, entre outras ${ }^{8}$. Sabe-se que a dor miofascial acometepessoas ativas, sendo a faixa etária entre 31 e 50 anos de idade a mais afetada ${ }^{8,9}$.

Dentre os tratamentos utilizados para a SDM, a liberação miofascial (LM), técnica da terapia manual utilizada na Fisioterapia, é a mais frequente, envolvendo especificamente forças mecânicas para manipular o complexo miofascial, com o objetivo de diminuir a dor e melhorar a função ${ }^{10}$. Como é frequentemente usada em condições ortopédicas, a LM é caracterizada como um "alongamento" gradativo do tecido mole realizado pelo fisioterapeuta, guiado inteiramente pelo feedback do paciente para determinar a direção, a força e a duração das manobras do tecido alvo ${ }^{11}$. No entanto, McKenney et al. ${ }^{12}$ ressaltam que a liberação miofascial também engloba pressão mínima aplicada ao tecido eassociada àliberação de pontos gatilhos.

Atualmente, devido a sua ampla aplicação no meio esportivo, vários estudos vêm discutindo a LM como uma possível técnica para minimizar sinais e sintomas musculoesqueléticos, entretanto, há controvérsias a respeito da eficácia da técnica. Segundo Kidd ${ }^{13}$, a LM depende muito da interação entre o terapeuta e o paciente para determinar a pressão realizada, o que dificulta sua padronização. Além disso, a subjetividade dessa interação deve ser abordada nas pesquisas envolvendo a técnica. Adicionalmente, Kidd $^{13}$ apontaque grande parte do efeito da liberação miofascial depende da habilidade do clínico e de sua capacidade tátil de sentir as mudanças teciduais, e que os efeitos biológicos do toque podem alterar a eficácia do tratamento. Sendo assim, esses autores discutem a confiabilidade entre avaliadores e a baixa qualidade metodológica das evidências.

Já Zugel et al. ${ }^{14}$ evidenciaram estudos em que a liberação miofascial produziu um aumento da perfusão sanguínea local e da excitabilidade corticoespinal, levando a uma melhora no deslizamento fascial, porém ressaltam que os mecanismos fisiológicos desta técnica ainda devem ser estudados, definindo o seu real efeito, já que estes podem surgir tanto da manipulação da fáscia como de tecidos adjacentes.

Dessa forma, vários métodos podem ser empregados para a LM. Porém, até o momento, não há um consenso sobre qual método seria mais eficaz e qual o protocolo a ser seguido. Além disso, pouco se sabe em relação aos efeitos fisiológicos e mecânicos da LM.

Levando em consideração o exposto acima, o presente estudo tem como objetivo verificar a eficiência da técnica de liberação miofascial em pacientes com sinais e sintomas causados pela "alteração fascial", sendo a população com características de síndrome de dor miofascial mais estudada para o uso de tal técnica.

\section{Métodos}

Foi utilizada como estratégia de busca para este estudo, consulta nas bases eletrônicas de dados US National Library of Medicine (PubMed), ScientificEletronic Library Online (SciELO)e Physiotherapy Evidence Database (PEDro),no período de 2008 a setembro de 2018, no idioma inglês e português, tendo como palavras-chaves: myofascial release, manual therapy, physical therapy, sendo o operador booleano "AND" utilizado nas combinações duas a duas das palavras-chaves.

Os critérios de inclusão para compor o presente estudo foram: 1) Classificação maior ou igual a $7 / 10$ na escala PEDro: 2) Ensaios Clínicos Randomizados (ECR); 3) tratamento utilizando técnicas diversas de liberação miofascial. A busca nas bases de dados foi realizada por dois pesquisadores, sendo a seleção dos artigos comparadas entre as duas buscas realizadas por eles. Caso houvesse divergência, na inclusão do artigo, um terceiro participante foi consultado.

\section{Resultados}

Os resultados obtidos na busca pelas palavras-chaves, combinadas duas a duas, resultaram em um total de 132 ensaios clínicos randomizados (ECR), dos quais 27 eram duplicatas. Foram eliminados quanto ao título da pesquisa 28 ECR, sendo estes eliminados quanto ao resumo - 11 ECR, eliminados por não alcançar pontuação igual ou superior a 7 na escala PEDro- 56 ECR, e por não se tratar de artigos no idioma inglês/português- 3 ECR, sendo, desta forma, considerados 7 ECR para inclusão no presente artigo (Figura 1).

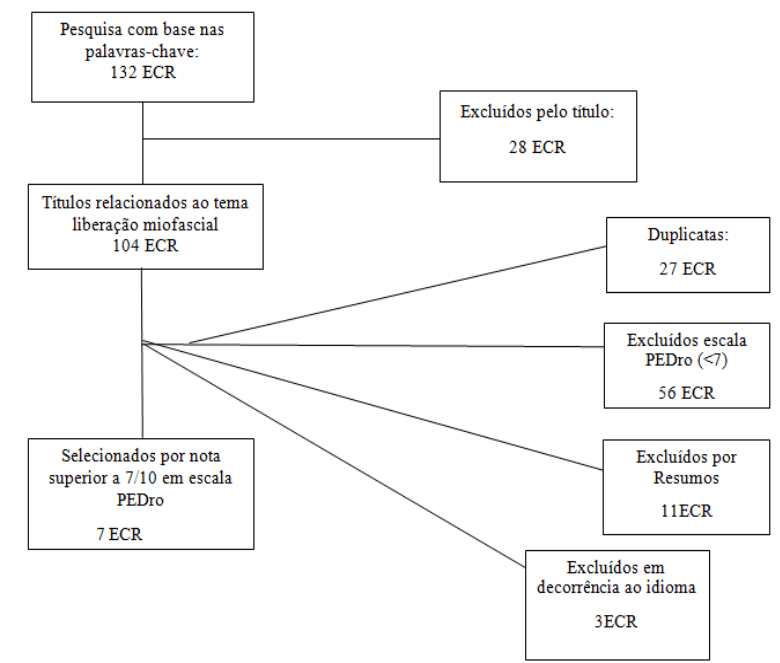

Figura 1. Fluxograma demonstrando seleção dos artigos, segundo os critérios de inclusão.

Os artigos selecionados para a discussão estão descritos na Tabela 1 conforme: autor e ano de publicação, classificação na escala PEDro, objetivo do estudo amostra, forma de intervenção, resultados e conclusão do estudo.

\section{Discussão}

De modo geral, a liberação miofascial, quando realizada por um profissional da área de reabilitação, se mostrou efetiva na redução da percepção de dor do paciente, levando a um aumento da amplitude de movimento (ADM) funcional, em todas as técnicas apresentadas nos artigos revisados. Entretanto, técnicas de autoaplicação, não apresentaram igual eficiência. Foram analisados artigos que realizaram a liberação miofascial 
de forma instrumental, por foam roll e FAT (Técnica Fascial de Abrasão) e de forma manual, com a aplicação de pressão em pinça, deslizamento profundo e ponto de compressão isquêmica.

Kim e Lee ${ }^{15}$ investigaram os efeitos da intervenção de liberação em pinça no músculo esternocledomastoideo (ECM) e pompagem na região cervical, em relação à tensão muscular e limiar de dor à pressão, em uma amostra pequena, composta por 17 sujeitos adultos. Com o uso da técnica de liberação em pinça do ECM, foi observadaredução na tensão muscular e um aumento no limiar de dor à pressão. Já com a técnica

Tabela 1

Descrição dos artigos selecionados após os critérios de inclusão. de pompagem, não foi observada alteração quanto tensão muscular e limiar de dor à pressão. Desta forma os autores concluíram que LM manual em pinça do ECM se mostrou efetiva na melhora do limiar de dor e tensão em ECM, principalmente quando comparados aos efeitos do grupo controle que realizaram a pompagem. Entretanto, apesar de alta qualidade metodológica, cabe ressaltar que o tamanho amostral poderia ter influenciado tal resultado. Também pode ter interferido neste resultado o fato de a amostra conter apenas 5 indivíduos do sexo masculino versus 12 do sexo feminino, visto que o limiar de dor à pressão poderia ter diferença entre os gêneros.

\begin{tabular}{|c|c|c|c|c|c|c|}
\hline $\begin{array}{l}\text { Autor e ano de } \\
\text { publicação }\end{array}$ & $\begin{array}{l}\text { Escala } \\
\text { PEDro }\end{array}$ & Objetivo & Amostra & Intervenção & Resultados & Conclusão \\
\hline $\begin{array}{l}\text { Kim e Lee } \\
(2018)^{15}\end{array}$ & $8 / 10$ & $\begin{array}{l}\text { Investigar os } \\
\text { efeitos da } \\
\text { intervenção de } \\
\text { liberação de } \\
\text { tecido mole em } \\
\text { relação à tensão } \\
\text { muscular e limiar } \\
\text { de dor à pressão }\end{array}$ & $\begin{array}{l}17 \text { sujeitos, } 5 \\
\text { homens e } 12 \\
\text { mulheres } \\
20-29 \text { anos com } \\
\text { queixas de } \\
\text { cervicalgia }\end{array}$ & $\begin{array}{l}\text { Liberação manual de } \\
\text { ECM com compressão } \\
\text { em pinça ou } \\
\text { deslizamento profundo. } \\
\text { Pompagem cervical. } \\
\text { 1x/semana por } 2 \\
\text { semanas }\end{array}$ & $\begin{array}{l}\text { Liberação em pinça do } \\
\text { ECM, foi observado } \\
\text { redução na tensão muscular } \\
\text { e um aumento no limiar de } \\
\text { dor a pressão. Já em } \\
\text { pompagem o músculo ECM } \\
\text { não apresentou alteração } \\
\text { quanto tensão muscular e } \\
\text { limiar de dor a pressão }\end{array}$ & $\begin{array}{l}\text { A liberação } \\
\text { muscular, em pinça, } \\
\text { do ECM se mostrou } \\
\text { efetiva na melhora } \\
\text { dor e tensão em } \\
\text { ECM }\end{array}$ \\
\hline $\begin{array}{l}\text { Rodriguez et al. } \\
(2018)^{16}\end{array}$ & $8 / 10$ & $\begin{array}{l}\text { Investigar a } \\
\text { eficácia da } \\
\text { terapia de } \\
\text { liberação } \\
\text { miofascial para } \\
\text { melhorar os } \\
\text { limiares de dor à } \\
\text { pressão e dor em } \\
\text { pacientes com } \\
\text { cervicalgia } \\
\text { mecânica }\end{array}$ & $\begin{array}{l}41 \text { sujeitos de } \\
\text { ambos os sexos. } \\
20-60 \text { anos com } \\
\text { diagnóstico de } \\
\text { cervicalgia } \\
\text { mecânica } \\
\text { inespecífica }\end{array}$ & $\begin{array}{l}\text { Liberação miofascial } \\
\text { com pressão profunda } \\
\text { progressiva } \\
\text { Intervenção } \\
\text { fisioterapêutica } \\
\text { multimodal com } \\
\text { aplicação de ultrassom, } \\
\text { TENS e massagem } \\
\text { clássica }\end{array}$ & $\begin{array}{l}\text { A liberação miofascial } \\
\text { apresentou uma melhora } \\
\text { significativa da dor quando } \\
\text { comparados aos sujeitos ao } \\
\text { programa multimodal, } \\
\text { resultado que se manteve } \\
\text { em reavaliação um mês } \\
\text { após a intervenção }\end{array}$ & $\begin{array}{l}\text { A liberação } \\
\text { miofascial se } \\
\text { mostrou superior a } \\
\text { uma intervenção } \\
\text { fisioterapêutica } \\
\text { multimodal na } \\
\text { melhora da dor }\end{array}$ \\
\hline $\begin{array}{l}\text { Arguisuelas et al. } \\
(2017)^{17}\end{array}$ & $9 / 10$ & $\begin{array}{l}\text { Investigar os } \\
\text { efeitos da } \\
\text { liberação } \\
\text { miofascial quanto } \\
\text { a dor, limitação } \\
\text { funcional e } \\
\text { cinesiofobia em } \\
\text { portadores de dor } \\
\text { lombar crônica }\end{array}$ & $\begin{array}{l}54 \text { sujeitos } 18-60 \\
\text { anos, ambos os } \\
\text { sexos com } \\
\text { lombalgia } \\
\text { inespecífica crônica }\end{array}$ & $\begin{array}{l}\text { Liberação miofascial } \\
\text { com ponto de pressão } \\
\text { progressiva e } \\
\text { deslizamento profundo } \\
\text { Liberação placebo com } \\
\text { deslizamento superficial } \\
\text { 2x/semana por } 3 \\
\text { semanas }\end{array}$ & $\begin{array}{l}\text { Os indivíduos do grupo } \\
\text { intervenção tiveram melhora } \\
\text { significativa em } \\
\text { questionários de limitação e } \\
\text { EVA }\end{array}$ & $\begin{array}{l}\text { A liberação } \\
\text { miofascial levou a } \\
\text { melhora funcional } \\
\text { em questionário e } \\
\text { melhora da dor }\end{array}$ \\
\hline $\begin{array}{l}\text { Rodriguez et al. } \\
(2016)^{18}\end{array}$ & $8 / 10$ & $\begin{array}{l}\text { Analisar os } \\
\text { efeitos da } \\
\text { liberação } \\
\text { miofascial versus } \\
\text { terapia manual } \\
\text { em um protocolo } \\
\text { multimodal para } \\
\text { cervicalgia } \\
\text { mecânica }\end{array}$ & $\begin{array}{l}\text { 59homens e } \\
\text { mulheres } \\
18-65 \text { anos, com } \\
\text { cervicalgia } \\
\text { mecânica irradiada } \\
\text { e pontuando } 10 \% \\
\text { no índice de } \\
\text { incapacidade }\end{array}$ & $\begin{array}{l}\text { TENS, termoterapia e } \\
\text { liberação miofascial com } \\
\text { pressão progressiva ou } \\
\text { protocolo de } \\
\text { alongamento, facilitação } \\
\text { neuromuscular } \\
\text { proprioceptiva e } \\
\text { mobilização cervical }\end{array}$ & $\begin{array}{l}\text { Após cinco sessões a } \\
\text { liberação miofascial se } \\
\text { mostrou mais efetiva quanto } \\
\text { a diminuição de dor e } \\
\text { posicionamento } \\
\text { craniovertebral, porém, } \\
\text { ambas mostraram efeito }\end{array}$ & $\begin{array}{l}\text { A liberação } \\
\text { miofascial foi mais } \\
\text { efetiva quanto a } \\
\text { melhora de ADM, } \\
\text { mostrando efeito em } \\
\text { reavaliação após } 5 \\
\text { sessões. Ambas as } \\
\text { terapias foram } \\
\text { efetivas na melhora } \\
\text { a longo prazo }\end{array}$ \\
\hline $\begin{array}{l}\text { Markovic } \\
2015^{19}\end{array}$ & $7 / 10$ & $\begin{array}{l}\text { Avaliar os efeitos } \\
\text { da liberação } \\
\text { miofascial } \\
\text { instrumental com } \\
\text { FAT tool ou } \\
\text { FoamRoll na } \\
\text { amplitude de } \\
\text { movimento }\end{array}$ & $\begin{array}{l}20 \text { jogadores de } \\
\text { futebol do sexo } \\
\text { masculinosaudáveis } \\
17-21 \text { anos }\end{array}$ & $\begin{array}{l}\text { Liberação em } \\
\text { foamrol/por duas séries } \\
\text { de } 1 \text { min/grupo } \\
\text { muscular. } \\
\text { Liberação em varredura } \\
\text { com o FAT por } 2 \\
\text { min/grupo muscular }\end{array}$ & $\begin{array}{l}\text { Ambas as técnicas levaram } \\
\text { a um aumento da ADM } \\
\text { ativa, porém o grupo FAT } \\
\text { teve o dobro de aumento de } \\
\text { ADM quando comparado ao } \\
\text { foamroll }\end{array}$ & $\begin{array}{l}\text { Ambas as técnicas } \\
\text { são efetivas para } \\
\text { ganho de ADM, } \\
\text { porém a FAT } \\
\text { demonstrou } \\
\text { superioridade } \\
\text { imediatamente e } 24 \mathrm{~h} \\
\text { após intervenção }\end{array}$ \\
\hline $\begin{array}{l}\text { Arroyo } \\
\text { et al. }(2008)^{20}\end{array}$ & $7 / 10$ & $\begin{array}{l}\text { Avaliar os efeitos } \\
\text { da liberação } \\
\text { miofascial sobre o } \\
\text { limiar nociceptivo } \\
\text { e recrutamento } \\
\text { neuromuscular } \\
\text { após exercício de } \\
\text { alta intensidade }\end{array}$ & $\begin{array}{l}62 \text { sujeitos de } \\
\text { ambos os sexos, } \\
\text { saudáveis, com } \\
18-26 \text { anos e ativos } \\
\text { (técnica utilizada } \\
\text { após a prática de } \\
\text { atividade física) }\end{array}$ & $\begin{array}{l}\text { Liberação miofascial } \\
\text { com pressão isquêmica } \\
\text { contínua e deslizamento } \\
\text { profundo em grandes } \\
\text { grupos musculares; } \\
\text { ultrassom simulado em } \\
\text { grupo controle }\end{array}$ & $\begin{array}{l}\text { O grupo submetido } \\
\text { àliberação miofascial } \\
\text { mostrou menor atividade } \\
\text { eletromiográfica e menor } \\
\text { tensão muscular quando } \\
\text { comparado ao grupo } \\
\text { placebo }\end{array}$ & $\begin{array}{l}\text { A liberação } \\
\text { miofascial levou a } \\
\text { um estado de } \\
\text { relaxamento } \\
\text { muscular pós } \\
\text { exercício com queda } \\
\text { de atividade em } \\
\text { EMG }\end{array}$ \\
\hline $\begin{array}{l}\text { Rezkallah } \\
\text { et al. }(2018)^{21}\end{array}$ & $7 / 10$ & $\begin{array}{l}\text { Comparar os } \\
\text { efeitos da técnica } \\
\text { de Mulligan } \\
\text { versus liberação } \\
\text { miofascial } \\
\text { associados a } \\
\text { exercícios na dor, } \\
\text { ADM e } \\
\text { incapacidade } \\
\text { funcional em } \\
\text { indivíduos com } \\
\text { cervicalgia }\end{array}$ & $\begin{array}{l}70 \text { sujeitos, ambos } \\
\text { os sexos, } 25-45 \\
\text { anos, com } \\
\text { cervicalgia } \\
\text { inespecífica }\end{array}$ & $\begin{array}{l}\text { Liberação miofascial } \\
\text { com deslizamento } \\
\text { profundo. } \\
\text { Mobilizaçãoantero- } \\
\text { superior a } 45^{\circ} \text { em } 3 \\
\text { séries de } 3-10 \\
\text { repetições. } \\
\text { Exercícios isométricos e } \\
\text { concêntricos e } \\
\text { alongamentos }\end{array}$ & $\begin{array}{l}\text { Houve uma melhora da dor } \\
\text { e ganho de ADM nos } \\
\text { grupos sujeitos a LMe } \\
\text { SNAG's. Também houve } \\
\text { melhora na funcionalidade } \\
\text { segundo questionário }\end{array}$ & $\begin{array}{l}\text { Ambas as técnicas } \\
\text { se mostraram } \\
\text { efetivas na melhora } \\
\text { da dor, } \\
\text { funcionalidade e } \\
\text { ADM, não se } \\
\text { demonstrando } \\
\text { superioridade entre } \\
\text { técnicas }\end{array}$ \\
\hline
\end{tabular}

ECM: músculo esternocleidomastóideo, TENS: Transcutaneous electrica Inervestimulation, EVA: Escala Visual Analógica, FAT: Fascial Abrasion Technique, ADM Amplitude de Movimento, EMG: Eletromiografia de Superfície, LM: Liberação Miofascial, SNAG: Sustained Natural Apophyseal GlideS.

Por outro lado, Rodriguez et al. ${ }^{16}$ investigaram a eficácia da LM na cervicalgia mecânicacom relação ao limiar de dor a pressão, sendo avaliado 41 sujeitos adultos. As intervenções utilizadas foram: a LM com pressão profunda progressiva, e intervenção fisioterapêutica multimodal com aplicação de ultrassom, TENS e massagem clássica. Ao final do tratamento foi observado que os pacientes submetidos à liberação miofascial tiveram melhora significativa da dor, quando comparados ao grupo submetido ao programa multimodal, sendo este resultado mantido após um mês. Dessa forma, os 
autores concluíram que a LM manual se mostrou eficaz e superior à intervenção multimodal, associando eletroterapia e massagem clássica, no alívio da dor em pacientes com cervicalgia mecânica inespecífica.

Diante do exposto, pode-se verificar que o uso da LM manual apresentou um efeito positivo relacionado ao alívio de dor, entretanto, cabe ressaltar que o manejoe a forma de execução da técnica manual é de difícil controle e padronização, a respeito da forçaexercida nas manobras de pressão profunda e deslizamento profundo, inserindo-se ainda outra variável, que é a velocidade de deslizamento manual.

Com relação à técnica de LM instrumental, Markovic ${ }^{19}$ avaliou os efeitos desta forma de liberação comparando a Fascial Abrasion Technique (FAT) e a Foam Roll (autoliberação), na amplitude de movimento de atletas. Foram avaliados apenas 20 jogadores de futebol, do sexo masculino, com idade entre 17 e 21 anos. A técnica de liberação em foam roll foi realizada em duas séries de 1 minuto por grupo muscular, e a liberação em varredura com o FAT durante 2 minutos por grupo muscular. As duas técnicas apresentaram melhora na ADM, entretanto, o grupo FAT apresentou um ganhoduas vezes superior, quando comparada àtécnica utilizando o foam roll.

Sendo assim, a LM instrumental, especialmente a FAT, demonstrou maior efetividade, visto que é aplicada pelo terapeuta com uma graduação manual de pressão em pontos específicos, tendo maior direcionamento e controle da força utilizada na liberação do que a técnica de foam roll, que exerce uma pressão muscular mais superficial. Além disso, cabe ressaltar que esta técnica é realizada na forma de autoliberação.

Rodriguez et al. ${ }^{18}$ analisaram os efeitos da LM manual com deslizamento profundo progressivo versus um programa fisioterapêutico multimodal que incluía a aplicação de TENS, termoterapia e terapia manual,associada à massagem clássica. Após cinco sessões, a LM se mostrou mais efetiva na diminuição de dor, porém, ambos os grupos apresentaram melhora, sendo que $43 \%$ dos indivíduos do grupo intervenção multimodal apresentaram melhora clínica frente a $90 \%$ dos indivíduos submetidos a LM. Cabe ressaltar que foram realizadas apenas cinco sessões.

Já Rezkallah et al. ${ }^{21}$ compararam os efeitos da técnica de mobilização articular pelo conceito Mulligan associados à exercícios versus LM, na dor, melhora de ADM e incapacidade funcional em indivíduos com cervicalgia mecânica inespecífica. A técnica de LM utilizada envolvia deslizamento profundo versus mobilização Mulligan em glide antero-superior associado a exercícios isométricos, concêntricos e alongamentos. Não foi observada superioridade para alguma das técnicas, pois ambas se mostraram efetivas na melhora da dor, funcionalidade e ADM.

Comparando os estudos de Rezkallah et al. ${ }^{21}$ e Rodriguez et al. ${ }^{16}$ observa se que métodos de terapia manual que geram mobilização tecidual e estrutural podem ser superiores a protocolos de eletroterapia e termoterapia. Além disso, Rezkallah et al. ${ }^{21}$ ressaltarama importância da associação de um programa de exercícios a longo prazo, que provavelmente contribuíram para a melhora do quadro geral do paciente, sendo importante quando se pensa em melhora funcional.

Arguisuelas et al. ${ }^{17}$, em um artigo com melhor qualidade metodológica, de acordo com a escala PEDro (9/10), apresentam bons resultados em relação à $L M$. Os autores investigaram os efeitos da LM na dor, limitação funcional e cinesiofobia em 54 pacientes com dor lombar crônica. A técnica de LM foi realizada com ponto de pressão progressiva e deslizamento profundo no grupo intervenção, sendo observada melhora significativa tanto na dor quanto incapacidade, em relação ao grupo controle, que realizou LM placebo.Estes autores afirmam ainda que a melhora clínica observada não poderia ser relacionada com o tecido miofascial, pois não foi avaliado a condição de tal tecido. Dessa forma, ensaios subsequentes serão necessários para avaliar o efeito da técnica no tecido.

Muitos artigos presentes na literatura abordam a eficácia da LM, porém, diante dos resultados favoráveis quanto a sua eficácia, novos ensaios clínicos deveriam abordar um comparativo entre técnicas de LM, verificando a eficácia e definindo assim qual o melhor protocolo. A grande dificuldade na criação de protocolos em LM se encontra na variabilidade de pressão e velocidades nas aplicações manuais. Porém, protocolos que estabelecessem a pressão estimada ideal seriam úteis para uma padronização da técnica e seus resultados. Também são necessários mais estudos que abordem a LM de um ponto de vista fisiológico, avaliando a sua influência nos tecidos, buscando explicações sobre quais estruturas são realmente afetadas pela $L M$, já que a pressão aplicada não atinge somente o nível fascial, mas também o nível muscular.

Ainda não existe um consenso sobre qual o momento deveria ser realizado a LM, anterior ou posterior a atividades físicas. Entretanto, Markovic ${ }^{19}$ acredita que a LM anterior a atividade, promoveria um ganho de ADM e, com isso, otimizaria o desempenho durante a prática. Ao contrário, Rezkallah et al. ${ }^{21}$ optaram por realizar a LM após as atividades físicas e também afirmam ter obtido bons resultados com relação à redução de dor e melhora de capacidade funcional. Diante dessa dualidade de opiniões, é necessário que haja um maior aprofundamento em pesquisas futuras em relação a este quesito.

Em relação ao meio esportivo, Le $\mathrm{Gal}^{22}$ afirma que a LM é considerada uma técnica alternativa, e comumente utilizadaanterior à prática esportiva, com o objetivo de promover ganho de ADM, redução de dores ou até mesmo uma forma de "aquecimento" para os atletas. A LM fornece vários efeitos benéficos, no entanto no meio esportivo, com o objetivo em se otimizar o tempo para realizar tal técnica, os atletas optam para a auto-liberação, por ser um método mais rápido e de fácil execução, já que os atletas usam a sua própria força corporal para execução.

De acordo com o exposto acima, podemos considerar que a LM apresenta grande relevância clínica, se mostrando efetiva na maioria das intervenções a respeito da melhora da dor. Porém, não foram realizadas medidas que demonstrassem mudanças estruturais. Dessa forma, não poderíamos afirmarque a eficácia da técnica de LM acontece em decorrência da alteração na organização tecidual, perfusão, redução de aderências e fibroses no tecido. Entretanto, apesar deste aspecto, a LM mostrou melhora de quadro de dor, incapacidade e ADM, demonstrando a necessidade de estudos nesta área.

Cabe ainda ressaltar que existem poucos estudos clínicos randomizados, pois a maior parte dos artigos encontrados nas bases de dados, devido à baixa qualidade metodológica dos artigos existentes (valores inferiores a 7 na escala PEDro), foram excluídos.Dessa forma, este aspecto deve ser ponderado na conclusão e na tomada de decisão clínica.

\section{Conclusão}

A técnica de liberação miofascial tem se mostrado uma boa estratégia no tratamento da dor miofascialem todas as suas formas de intervenção, porém, as técnicas de autoliberação apresentaram menoreficiência quando comparada as liberações realizadas por profissionais. Tanto as intervenções manuais quanto as realizadas por meio instrumental demonstraram bons resultados. Contudo, devem ser realizados mais estudos de alta qualidade metodológica para definir os reais efeitos fisiológicos e clínicos dastécnicas em questão.

\section{Referências}

1. Adstrum S, Hedley G, Schleip R, Stecco C, Yucesoy CA.Defining the fascial system. J Bodyw Mov Ther2017; 21:173-177.

2. Schleip R, Jager H, Klingler W. What is 'fascia'? A review of different nomenclatures. J Bodyw Mov Ther 2012; 16(4):496-502.

3. Chaitow L. Terapia Manual para disfunção fascial. Artmed, 2017.

4. Moccia D, Nackashi AA, Schilling R, Ward PJ. Fascial bundles of the infraspinatus fascia: anatomy, function and clinical considerations. J Anat 2016; 228(1):176-183.

5. Zullo A, Mancini FP, Scleip R, Wearing S, Yahia L, Klingler W. The interplay between fascia, skeletal muscle, nerves, adipose tissue, inflammation and mechanical stress in musculo-fascial regeneration. $J$ Geront Geriatrics 2017; 65:271-283.

6. Langevin HM In: Audette JF, ailey A (eds). Integrative pain medicine. Humana Press, New York.

7. Bennett RM, Friend $R$, Marcus $D$, Bernstein $C$, Han BK, Yachoui $R$, Deodhar A, Kaell A Bonafede P Chino A Jones KD. Criteria for the Diagnosis of Fibromyalgia: Validation of the Modified 2010 Preliminary Diagnosis of Fibromyalgia: Validation of the Modified 2010 Preliminary
American College of Rheumatology Criteria and the Development of Alternative Criteria. ArthritisCareRes 2014; 66:1364-1373.

8. Bigongiari A, Franciulli PM, Andrade e Souza F, Araujo RC. Análise da atividade eletromiográfica de superfície de pontos gatilhos miofasciais. Rev. Bras. Reumatol 2008; 48(6):319-324.

9. Teixeira MJ. Dor, epidemiologia, fisiopatologia, avaliação, síndromes dolorosas e tratamento. São Paulo: Grupo Editorial Moreira Jr; 2001.

10. Barnes MF. The basic science of myofascial release: morphologic change in connective tissue. J Bodyw MovTher 1997;1(4):231-238. 
11. Manheim CJ. The myofascial release manual. Slack incorporated, $4^{\text {th }}$ edition; 2008.

12. McKenney K, Elder AS, Elder C, Hutchins A. Myofascial release as a treatment for orthopaedic conditions: a systematic review. J Athl Train 2013;48(4):522-7.

13. Kidd RF. Why myofascial release will never be evidence-based. Int. Musculoskelet. Med. 2013; 31(2): 55-56

14. Zügel M, Maganaris CN, Wilke J, Jurkat-Rott $K$, Klingler W, Wearing SC, Findley T, Barbe MF, Steinacker JM, Vleeming A, Bloch W, Schleip R, Hodges PW.Fascial tissue research in sports medicine: from molecules to tissue adaptation, injury and diagnostics: consensus statement. $\mathrm{Br} \mathrm{J}$ Sports Med2018; 52(23):1497

15. Kim SJ, Lee JH. Effects of sternocleidomastoid muscle and suboccipital muscle soft tissue release on muscle hardness and pressure pain of the sternocleidomastoid muscle and upper trapezius muscle in smartphone users with latent trigger points. Medicine 2018;97(36):e12133.

16. Rodriguez-Huguet M, Gil-Salú JL, Rodrigues-Huguet P, Cabrera-Afonso JR, Lomas-Vegas R. Effects of Myofascial Release on Pressure Pain Thresholds in Patients With Neck Pain: A Single-Blind Randomized Thresholds in Patients With Neck Pain: A Single-Blind
Controlled Trial. Am J Phys Med Rehabil 2018; 97(1):16-22.

17. Arguisuelas MD, Lison JF, Sanches-zuriaga D, Martinez-Hurtado I, Domenech-Fernandez J. Effects of Myofascial Release in
Nonspecific Chronic Low Back Pain: A Randomized Clinical Trial. Spine 2017; 42(9):627-634

18. Rodriguez-Fuentes I, De Toro FJ, Rodrigues-Fuentes G, de Oliveria IM Meijide-Failde R, Fuentes-Boguete IM. Myofascial Release Therapy in the Treatment of Occupational Mechanical Neck Pain: A Randomized Parallel Group Study. Am JPhys Med Rehabil 2016;95(7):507-15.

19. Markovic $G$. Acute effects of instrument assisted soft tissue mobilization vs. foam rolling on knee and hip range of motion in soccer players. JBodywMovTher 2015; 19(4):690-696.

20. Arroyo-Morales M, Olea N, Martinez MM, Hidalgo-Lozano A, RuizRodrigues C, Díaz-Rodrigues L.Psychophysiological Effects of MassageMyofascial Release After Exercise: A Randomized Sham-Control Study. J Altern Complement Med 2008; 14(10):1223-1229.

21. Rezkallah S, Abdullah GA. Comparison between sustained natural apophyseal glides (SNAG's) and myofascial release techniques combined with exercises in non specific neck pain. Physiother Pract Res 2018; 39(2):135-145.

22. Le Gal J, Begon M, Gillet B, Rogowski I.Effects of Self-Myofascial Release on Shoulder Function and Perception in Adolescent Tennis Players. J Sport Rehabil 2018; 27(6):530-535. 